\title{
Metformin's contraindications: needed for now
}

\author{
I. George Fantus
}

ß See related article page 502

This commentary reflects George Fantus' viewpoint on whether the current contraindications to metformin therapy are warranted for certain patients with type 2 diabetes. After he was asked to peer review the unsolicited commentary by James McCormack and coauthors on the topic (see page 502), he weighed some of the same evidence and reached a different conclusion. We thought you might find it as interesting as we did to read 2 careful considerations of the same clinical question that reach different answers. Evidence, at least for this issue, is in the eye of the beholder. - CMAJ

A ppropriate drug prescribing is an ongoing challenge and may at times generate controversy. Whether we have adequate and reliable data to alter our prescribing of the antihyperglycemic agent metformin for type 2 diabetes mellitus is addressed in this commentary.

Metformin is currently recommended as an agent of choice for the treatment of type 2 diabetes, particularly in obese patients. ${ }^{1,2}$ Its effectiveness in lowering blood glucose concentrations as a consequence of inhibiting hepatic glucose production and insulin resistance is well established., ${ }^{3,4}$ More recent data from the UK Prospective Diabetes Study (UKPDS) indicated that obese patients with newly diagnosed type 2 diabetes who received metformin had a significant ( $7 \%$ absolute) reduction in the rate of diabetes-related death and a $10 \%$ reduction in overall mortality after 10 years of follow-up compared with patients following a prescribed diet only and those given a sulfonylurea, despite similar glycemic control in the latter group. ${ }^{5}$ This apparent protection against vascular complications independent of glucose control presents a novel attribute to an oral antihyperglycemic agent and has stimulated the prescribing of metformin.

As with all pharmacologic agents, there are limitations and contraindications to the use of metformin. ${ }^{6}$ Although gastrointestinal side effects may render the drug intolerable or limit the dose, the main concern with metformin is lactic acidosis. The drug is a biguanide. In the 1960s and early 1970s, other biguanides (phenformin and buformin) were available to treat type 2 diabetes. Despite their efficacy as glucose-lowering agents, they were removed from the market because their use was associated with an unacceptably high incidence of lactic acidosis, a condition with a $40 \%-50 \%$ death rate. ${ }^{7,8}$ The safety record of metformin is much better. Monitoring in Canada from 1972 to 1983 revealed only 36 cases of lactic acidosis after $56000 \mathrm{pa}$ - tient-years of use. ${ }^{9}$ More recent retrospective analyses have reported that lactic acidosis occurs as frequently among patients with type 2 diabetes not taking metformin as among those taking the drug. ${ }^{10}$ These findings have caused some clinicians to question whether metformin can cause or contribute to lactic acidosis ${ }^{11}$ and whether it is necessary to maintain current contraindications to its use (severe liver disease, congestive heart failure, age $\geq 80$ years and renal impairment). ${ }^{4,6}$

From our knowledge of the mechanism of action of metformin, increased lactic acid production is possible. Similar to all biguanides, metformin acts in the mitochondria to inhibit either the Krebs (tricarboxylic acid) cycle ${ }^{12}$ or oxidative phosphorylation, ${ }^{13}$ or both. Recent discoveries indicate that the enzyme AMP (adenosine monophosphate)-kinase is activated..$^{14}$ AMP-kinase is activated under physiologic conditions by elevation in cellular AMP concentration, which occurs during exercise or fasting. The degree of mitochondrial inhibition by metformin may be less than that by the other biguanides, but the most important factor that accounts for metformin's relative safety is that, in contrast to phenformin, it does not require hepatic metabolism and instead is excreted unchanged by the kidney. Thus, renal function is a key determinant of safety: although lactic acidosis has occurred because of ingestion of toxic amounts of metformin in a few cases ${ }^{16}$ most cases of metformin-associated lactic acidosis involved patients with impaired renal function. ${ }^{9,15}$ The current contraindications revolve around the concerns of clearance of lactate (liver), predisposition to lactic acidosis (congestive heart failure, severe liver disease, uremia) and renal impairment (elevated serum creatinine level, congestive heart failure and advanced age). Are the safety record and potential vascular protection reasons enough to remove or modify these contraindications to extend metformin's use in type 2 diabetes?

The first issue to address in order to answer this question is whether patients with type 2 diabetes who receive metformin despite having contraindications to it are at increased risk of lactic acidosis. Such a study has not been performed and would probably be ethically difficult given the high mortality rate associated with lactic acidosis. Also, because lactic acidosis is uncommon, obtaining reliable data would require a large study population. Thus, we are left with retrospective reports of outcomes among patients given metformin in a "contraindicated" setting. Another 
caveat of these observational studies is that clinicians would likely have judged the contraindication to be relative rather than absolute. For example, an 81-year-old patient with type 2 diabetes and documented normal renal function or a patient with mild and stable congestive heart failure could have received the drug. Despite these limitations, a study from Scotland reported 1 case of lactic acidosis among 1847 patients given metformin over 30 months, $25 \%$ of whom had contraindications. ${ }^{17}$ This translates to an annual rate of lactic acidosis of 1 per all 4600 patients taking metformin but 1 per 1155 patients given the drug "inappropriately." (The patient in whom lactic acidosis developed had renal impairment [creatinine level $152 \mathrm{mmol} / \mathrm{L}]$.) If we assume that the prevalence of diabetes in Canada is $6 \%$ in a population of about 30 million and that $90 \%$ of these cases are type 2 diabetes, a total of 1.8 million Canadians are affected. Assuming that 50\%-60\% (about 1 million) are prescribed oral therapy and, as indicated in the Scottish Study, $25 \%$ of these $(250000)$ have some contraindication to metformin but will be administered the drug in any case, we may have 225-250 new cases of lactic acidosis in Canada annually. With a $40 \%-50 \%$ mortality rate, this would be unacceptable. Although these "back of the envelope" calculations may overestimate the risk, it is important to emphasize, as Salpeter and coauthors point out, ${ }^{18}$ that the conclusion about the safety of metformin is based on its appropriate use and adherence to the contraindications.

In addition to an assessment of risk, it is necessary to consider the potential benefits of metformin used in a contraindicated setting. Is there an alternative agent? Lowering of blood glucose concentrations in patients with contraindications to metformin can be achieved through the use of either another orally administered antihyperglycemic agent or, usually most effectively, insulin. Although psychological and human-resource barriers may exist to the initiation of insulin therapy, these can almost always be overcome. We are thus left with the possible nonglycemic benefit of vascular protection as the only rationale for prescribing metformin in the setting of contraindications. In the UKPDS, this effect was observed over 10 years among obese subjects with newly diagnosed type 2 diabetes who had no renal impairment or congestive heart failure. ${ }^{5}$ The first concern, therefore, is whether these results are applicable to patients with type 2 diabetes who have contraindications. Those with congestive heart failure and moderate to severe renal failure generally have a poorer prognosis. In addition to the question of applicability is a second concern about the primary data, which has been addressed in some detail. ${ }^{19}$ Nathan pointed out that, in the UKPDS, the metformin-treated group was compared primarily with a control (non-intensively treated) diet group, not with the other intensively treated groups. In the secondary analysis, which supported the benefit of metformin over the other intensive treatments, the obese subjects given sulfonylureas and insulin unexpectedly did not show an improved outcome over the dietonly group. ${ }^{5}$ This finding was in contrast to the overall findings of the UKPDS when obese and lean subjects were combined. Another unexplained observation in the UK study was that the subjects who received metformin early, as an add-on to their sulfonylurea therapy, not only showed no protective benefit of metformin, they also had significantly higher rates of diabetes-related death and all-cause mortality than the group who had sulfonylurea therapy alone. ${ }^{5,19}$ Despite post hoc statistical analysis to "disprove" the latter adverse effect of metformin, this observation remains troubling, since the majority of patients require combination therapy. A third confounding factor is that our goals for cardiovascular risk reduction, in terms of blood pressure and low-density lipoprotein cholesterol targets, are far more aggressive today than they were in the mid-1970s, when the UKPDS was designed. We do not know whether the benefit of metformin would be apparent in the context of current treatment guidelines. Given the uncertainty of the primary data, particularly lacking as they apply to patients with contraindications to metformin, there is currently no sound evidence to support the conclusion that the benefits of metformin will outweigh its risks in patients with contraindications to its use.

The position statement of the American Diabetes Association on the implications of the UKPDS reflects these concerns and concludes that "until such a trial is concluded [i.e., a prospective, randomized, placebo-controlled trial], we do not recommend any change in the current guidelines for the use of metformin as monotherapy or in combination with sulfonylurea drugs." ${ }^{20}$ Finally, it should be noted from anecdoctal experience combined with retrospective reports of metformin use ${ }^{7}$ that many physicians adhere to the contraindications "loosely." If these were removed or made less stringent, we would possibly, even 
probably, see an increase in the incidence of lactic acidosis. As discussed above, it is difficult to predict with precision the expected morbidity and mortality. However, even 1 or 2 publicized cases would generate fear among all our patients and could discourage metformin use in appropriate settings. Thus, until a favourable risk-benefit ratio is documented from clinical trial data, the maintenance of currently accepted contraindications would appear to be prudent.

This article has been peer reviewed.

George Fantus is with the Division of Endocrinology, Department of Medicine, Mount Sinai Hospital and University Health Network, University of Toronto, Toronto, Ont

Competing interests: George Fantus received an honorarium for a lecture from Bristol Myers Squibb, manufacturer and distributor of metformin in the United States.

\section{References}

1. Canadian Diabetes Association Clinical Practice Guidelines Expert Committee. Canadian Diabetes Association 2003 clinical practice guidelines for the prevention and management of diabetes in Canada. Can 7 Diabetes 2003 27(Suppl 2):S1-152. Available: www.diabetes.ca/cpg2003/chapters.aspx (accessed 2005 July 26).

2. Cheng AYY, Fantus IG. Oral antihyperglycemic therapy for type 2 diabetes mellitus. CMA7 2005;172(2):213-26.

3. DeFronzo RA, Goodman AM; Multicenter Metformin Study Group. Efficacy of metformin in patients with non-insulin dependent diabetes mellitus. $N$ Engl 7 Med 1995;333:541-49.

4. Kirpichnikov D, McFarlane SI, Sowers JR. Metformin: an update. Ann Intern Med 2002;237:25-33.

5. UK Prospective Diabetes Study (UKPDS) Group. Effect of intensive bloodglucose control with metformin on complications in overweight patients with type 2 diabetes (UKPDS 34) [published erratum in Lancet 1998;352:1557]. Lancet 1998;352:854-65.
6. Bailey CJ, Turner RC. Metformin. N Engl 7 Med 1996;334:574-79.

7. Misbin RI. The phantom of lactic acidosis due to metformin in patients with diabetes. Diabetes Care 2004;27:1791-3.

8. Lactic Acidosis Study Group. Natural history and course of acquired lactic acidosis in adults. Am 7 Med 1994;97:47-54

9. Lucis OJ. The status of metformin in Canada. CMA7 1983;128(1):24-6.

10. Salpeter S, Greyber E, Pasternak G, Salpeter E. Risk of fatal and non-fatal lactic acidosis with metformin in type 2 diabetes [Cochrane review]. In: The Cochrane Library; Issue 4, 2004, Oxford: Update Software.

11. Stades AME, Heikens JT, Erkelens DW, Holleman F, Hoekstra JBL. Metformin and lactic acidosis: Cause or coincidence? A review of case reports. 7 Intern Med 2004;255:179-87.

12. Steiner DF, Williams RH. Respiratory inhibition and hypoglycemia by biguanides and decamethylenediguanidine. Biochim Biophys Acta 1958;30:329-40.

13. Owen M.R., Doran E., Halestrap A.P. Evidence that metformin exerts its anti-diabetic effects through inhibition of complex 1 of the mitochondrial respiratory chain. Biochem 7 2000;348:607-14.

14. Musi N, Hirshman MF, Nygren J, Svanfeldt M, Bavenholm P, Rooyackers O et al. Metformin increases AMP-activated protein kinase activity in skeletal muscle of subjects with type 2 diabetes. Diabetes 2002;51:2074-81.

15. Misbin RI, Green L, Stadel BV, Gueriguian JL, Gubbi A, Fleming GA. Lactic acidosis in patients with diabetes treated with metformin [letter]. $N$ Engl $\mathcal{F}$ Med 1998;338:265-6.

16. Chang CT, Chen YC, Fang JT, Huang CC. Metformin-associated lactic acidosis: case reports and literature review. 7 Nephrol 2002;15:398-402.

17. Emslie-Smith AM, Boyle DIR, Evans JMM, Sullivan F, Morris AD. Contraindications to metformin therapy in patients with Type 2 diabetes - a population-based study of adherence to prescribing guidelines. Diabet Med 2001;18:483-8.

18. Salpeter S, Greyber E, Pasternak G, Salpeter E. Risk of fatal and non-fatal lactic acidosis with metformin in type 2 diabetes. Arch Intern Med 2003;163. 2594-602.

19. Nathan DM. Some answers, more controversy, from UKPDS [editorial]. Lancet 1998;352:832-3.

20. American Diabetes Association. Implications of the United Kingdom Prospective Diabetes Study [position statement]. Diabetes Care 2003;26(Suppl 1):S28-32

Correspondence to: Dr. I. George Fantus, Leadership Sinai Centre for Diabetes, Mount Sinai Hospital, Rm. 5028, 5th floor, Lebovic Building, 600 University Ave., Toronto ON M5G 1X5

\section{Clinical trial registration}

CMAJ will consider clinical trials for publication only if they have been registered in a publicly accessible clinical trials registry before the enrolment of the first patient. This policy applies to trials that start recruiting on or after July 1, 2005. For trials that began enrolment before this date, registration is required by Sept. 13, 2005. The criteria for acceptable registration are described in CMAJ (2005;172[13]:1700-2). 